\title{
Calcified Urachal Cancer Managed by Partial Cystectomy: A Case Report
}

\author{
Taras Hladun' \\ Jakub Ratajczak' \\ Maciej Salagierski \\ 'Urology Department, Regional \\ Specialized Hospital in Nowa Sól, Nowa \\ Sól, 67-100, Poland; '²Department of \\ Urology, Collegium Medicum, University \\ of Zielona Góra, Zielona Góra, 65-046, \\ Poland
}

\begin{abstract}
This report presents the case of a rare and aggressive cancer originating from the urachus in a 73-year-old female. After 12 years of observation due to a cystic lesion in the bladder dome, the patient rapidly developed haematuria and mucinuria. The use of multiple diagnostic measures suggested urachal malignancy. Partial cystectomy and urachal excision along with pelvic lymphadenectomy were performed. Urachal adenocarcinoma with negative surgical margins and lymph nodes was reported in pathology. Follow-up after 12 months did not reveal any cancer relapse. Epidemiological, clinical and therapeutic features of this disease are also discussed.
\end{abstract}

Keywords: urachus, adenocarcinoma, haematuria, mucinuria, cystectomy

\section{Introduction}

In foetal development, the urachus connects the bladder to the allantois. After closure of the urachal canal, it forms the median umbilical ligament, stretching from the dome of the bladder to the umbilicus in the midline and extraperitoneally within the space of Retzius. Research shows that the canal fails to close in $1.5 \%$ of newborns. ${ }^{1}$ Incomplete closure manifests as persistent urachus, urachal cyst or urachal fistulae. The longstanding inflammatory process within these malformations is the main factor that leads to dysplasia and finally to cancer. Genetic predisposition, familial clustering and environmental factors are not known to cause urachal cancer. Here, we report a case of clinical management of long-developing urachal cancer.

\section{Case Presentation}

A 73-year-old woman with dysuria, intermittent haematuria and mucous discharge in urine was referred to the urology department. The patient had been under outpatient observation for 12 years owing to a $2.3 \mathrm{~cm}$ cyst of the bladder. The lesion was located at the dome of the bladder wall, without connection to the bladder lumen, which was confirmed in the initial computed tomography (CT) scan. Every year, the patient underwent ultrasound examination. Clinical laboratory tests had been performed with higher frequency in the last 5 years of observation owing to higher rates of uncomplicated urinary tract infections. Radiological findings gathered over the years revealed the slow growth of the lesion with close proximity to the intestinal wall, and finally new calcifications and probable connection to the bladder lumen. Considering the radiological findings, the following were included in the differential diagnosis: urachal cyst, vesical diverticula or bowel neoplasia. No pathological findings were obtained from colonoscopy examination.
Correspondence: Maciej Salagiersk Department of Urology, Collegium Medicum, University of Zielona Góra Zyty 26, Zielona Góra, 65-046, Poland Tel +48504 174253

Email m.salagierski@cm.uz.zgora.pl 
After the patient developed haematuria and mucous discharge in urine, urgent CT (Figures 1 and 2) and magnetic resonance imaging (MRI) (Figure 3) scans showed a $4.2 \mathrm{~cm}$ calcified, cystic lesion connected to the lumen of the bladder. In a district hospital, the patient underwent a cystoscopy and colonoscopy, owing to a suspected vesicointestinal fistula. The patient then underwent bladder tumour electroresection in a secondary-level hospital. The histopathologist reported bladder mucosa inflammation and atypical cells originating from bowel or urachal tissue. After multidisciplinary consultation, it was decided to perform open diagnostic laparotomy with possible extended bladder or bowel surgery with faecal and urinary diversions. During the operation, a calcified tumour originating from the urachal remnant was discovered. The tumour, with a size of $5 \times 4 \mathrm{~cm}$, infiltrated the bladder dome. Both large and small intestines were intact. The urachal remnant excision was performed "en bloc" with partial cystectomy and pelvic lymphadenectomy, preserving the bladder trigone,

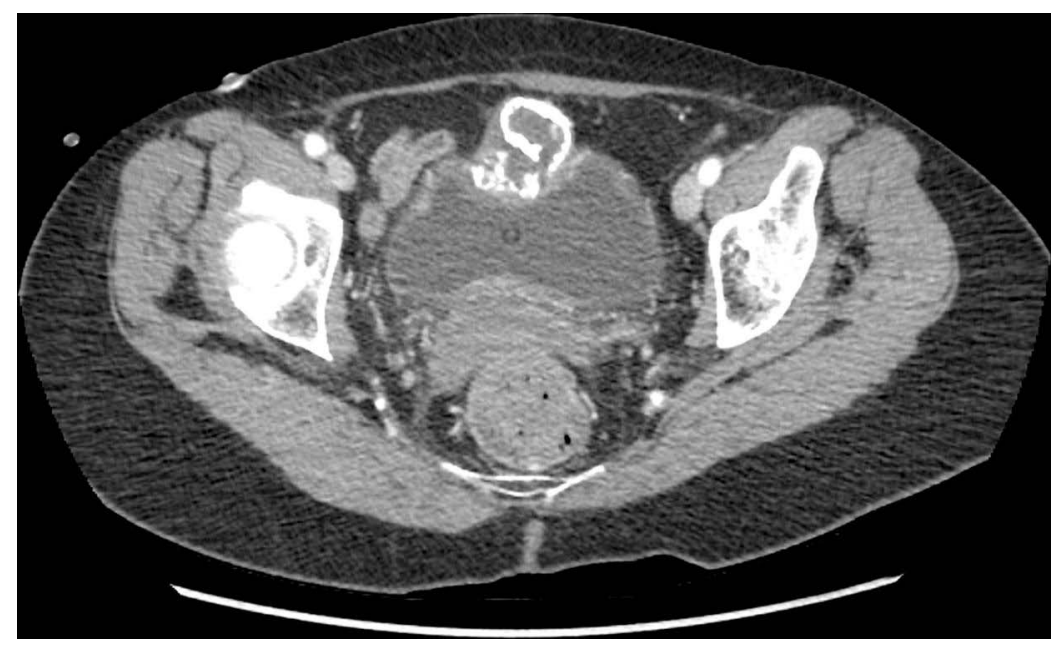

Figure I CT scan showing the calcified lesion arising within the bladder wall.

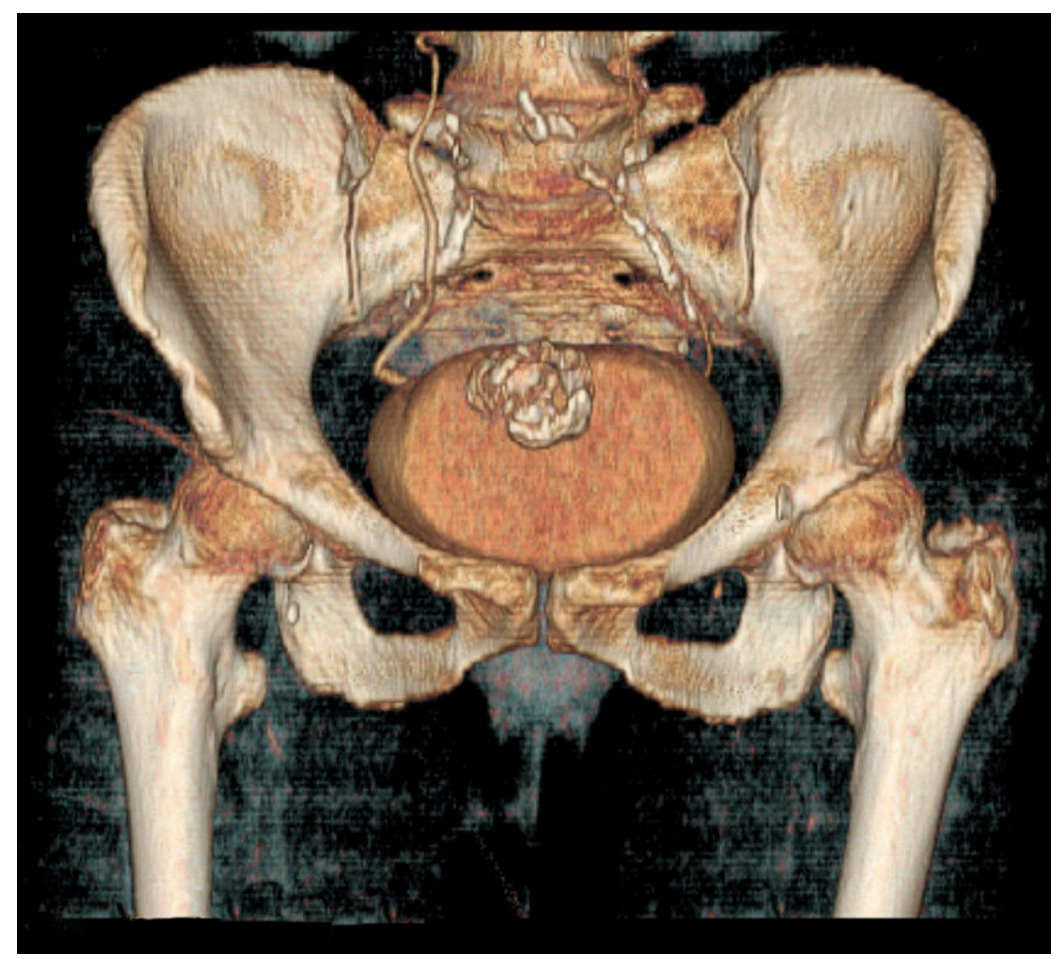

Figure 2 3D CT reconstruction showing the topographic contiguity of the calcified lesion and bladder wall. 


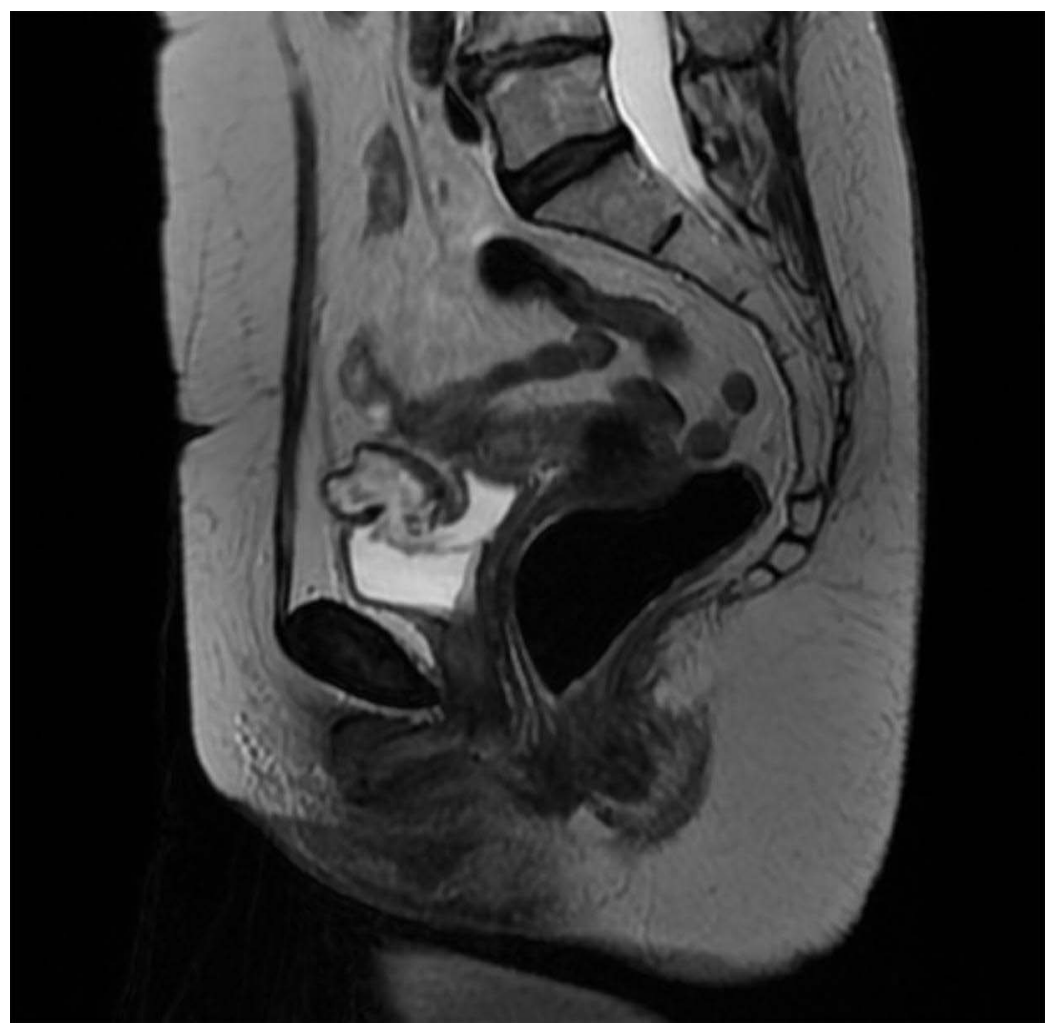

Figure 3 MRI scan showing the connection of the calcified cystic lesion with the bladder lumen.

lateral and posterior walls. No bladder augmentation was necessary as its residual volume was around $200 \mathrm{~mL}$. Twolayer sutures with Vicryl 3/0 and 2/0 were performed to close the remaining bladder. The patient was discharged from the department with an indwelling urethral catheter for 2 weeks. Pathology reported urachal adenocarcinoma with negative bladder margins and lymph nodes, resulting in no further chemotherapy. Follow-up (after 12 months) showed no cancer relapse. The patient has provided written informed consent for the case details to be published.

\section{Discussion}

Urachal cancer is relatively rare and occurs in $0.2 \%$ of bladder neoplasms. ${ }^{2}$ The disease remains asymptomatic in the early stages, and thus nearly $70-90 \%$ of tumours are diagnosed after extension to the bladder wall (Sheldon III stage). Typical manifestations are haematuria (85\%) and mucinuria (17\%), whereas abdominal pain, palpable mass in the lower abdomen or mucous discharge are less common. ${ }^{3}$ In cystoscopy, the tumour most often presents as a polyp or ulceration located in the dome or anterior wall of the bladder. Initial ultrasound evaluation may reveal heterogeneous soft tissue mass containing fluid or calcifications. Further CT or MRI scans facilitate proper differentiation between bladder and urachal malignancy. In CT scans, $60 \%$ of urachal carcinoma cases present low-attenuation components, suggesting mucin content, and in $50-70 \%$ of cases calcifications are observed. Some scientific researchers ${ }^{6,7}$ suggest that calcifications in $\mathrm{CT}$ are considered nearly pathognomonic for urachal adenocarcinoma. Adenocarcinoma represents the vast majority (65-82\%) of urachal tumours. ${ }^{5}$ Histopathological examination of the bladder lesion with immunohistochemistry differentiates between primary bladder adenocarcinoma, if positive for keratin 7 (CK7) and 20 (CK20), in contrast to adenocarcinoma of the colon, which contains only CK20. The primary method of treatment is partial cystectomy with optional umbilicotomy or radical cystectomy. Both methods provide similar oncological results. However, bladder-sparing resection of the organ provides better quality of life. Lymphadenectomy improves survival rates, because positive regional lymph nodes have a similar impact to the presence of distant metastases. Late diagnosis of the tumour implicates a high incidence of distant metastases, exceeding $20 \%$, whereas local lymph-node invasion was reported in $17 \%$ of cases. Median overall survival in the Dutch cohort analysed by Bruins et al was 48 months. Relative 5-year survival (RS) in patients with non-metastatic disease (Sheldon I-III) was 61\%, whereas in metastatic 
disease (stage IV) it dropped to only $15 \% .^{2}$ Although chemotherapy with 5-fluorouracil (5-FU) or cisplatin has been shown to be effective, high-quality data are unavailable, mainly because of the rarity of the disease. Meta-analysis by Szarvas et al concluded that 5-FU-based chemotherapy resulted in a better radiographic response than a cisplatinbased regimen; however, in metastatic disease, synergy of cisplatin and 5-FU was the most effective. Targeted molecular therapy will provide a promising alternative to chemotherapy in the future. ${ }^{4}$ No evidence of clinical benefit from radiotherapy in the management of urachal adenocarcinoma has been reported in the literature.

\section{Conclusion}

Despite the rarity and diverse clinical presentations of urachal adenocarcinoma, every physician must be attentive to patients with haematuria, mucinuria, suprapubic pain and imaging findings of thickening of the vesical dome. The main treatment for urachal adenocarcinoma is surgical resection. Chemotherapy has been reserved for cases of metastatic disease or local disease relapse. Timely surveillance can help to improve outcomes and survival rates.

\section{Informed Consent}

Written informed consent was received from the patient.

\section{Disclosure}

The authors declare that they have no competing interests.

\section{References}

1. Gleason JM, Bowlin PR, Bagli DJ, et al. A comprehensive review of pediatric urachal anomalies and predictive analysis for adult urachal adenocarcinoma. J Urol. 2015;193(2):632-636. PMID: 25219697. doi:10.1016/j.juro.2014.09.004

2. Bruins HM, Visser O, Ploeg M, et al. The clinical epidemiology of urachal carcinoma: results of a large, population based study. J Urol. 2012;188(4):1102-1107. PMID: 22901574. doi:10.1016/j. juro.2012.06.020

3. Molina JR, Quevedo JF, Furth AF, et al. Predictors of survival from urachal cancer: a Mayo Clinic study of 49 cases. Cancer. 2007;110 (11):2434-2440. PMID: 17932892. doi:10.1002/cncr.23070

4. Szarvas T, Módos O, Niedworok C, et al. Clinical, prognostic, and therapeutic aspects of urachal carcinoma-a comprehensive review with meta-analysis of 1010 cases. Urol Oncol. 2016;34 (9):388-398. PMID: 27267737. doi:10.1016/j.urolonc.2016. 04.012

5. Hager T, Kraywinkel K, Szarvas T, et al. Urachal cancer in Germany and the USA: an RKI/SEER Population-Based Comparison Study. Urol Int. 2020;104(9-10):803-809. PMID: 32784300. doi:10.1159/ 000509481

6. Yu J-S, Kim KW, Lee H-J, et al. Urachal remnant diseases: spectrum of CT and US findings. Radiographics. 2001;21(2):451. PMID: 11259707. doi:10.1148/radiographics.21.2.g01mr02451

7. Ashley RA, Inman BA, Sebo TJ, et al. Urachal carcinoma: clinicopathologic features and long-term outcomes of an aggressive malignancy. Cancer. 2006;107(4):712-720. PMID: 16826585. doi: $10.1002 /$ cncr. 22060
Research and Reports in Urology

\section{Publish your work in this journal}

Research and Reports in Urology is an international, peer-reviewed, open access journal publishing original research, reports, editorials, reviews and commentaries on all aspects of adult and pediatric urology in the clinic and laboratory including the following topics: Pathology, pathophysiology of urological disease; Investigation and

\section{Dovepress}

treatment of urological disease; Pharmacology of drugs used for the treatment of urological disease. The manuscript management system is completely online and includes a very quick and fair peer-review system, which is all easy to use. Visit http://www.dovepress.com/ testimonials.php to read real quotes from published authors. 